\title{
DEPRESIÓN, ANSIEDAD Y ESTRÉS EN ESTUDIANTES DE NUEVO INGRESO A LA EDUCACIÓN SUPERIOR.
}

DEPRESSION, ANXIETY AND STRESS IN NEW STUDENTS ENTERING TO HIGHER EDUCATION

Tijerina González Liliana Zandra*, González Guevara Erika*, Gómez Nava Marisol*, Cisneros Estala

Martha Aidee*, Rodríguez García Karla Yadira*, Ramos Peña Esteban Gilberto*.

*Universidad Autónoma de Nuevo León, Facultad de Salud Pública y Nutrición, México.

Citation: Tijerina González L.Z., González Guevara E., Gómez Nava M., Cisneros Estala M.A., Rodríguez García K.Y., Ramos Peña E.G. (2018) Depresión, ansiedad y estrés en estudiantes de nuevo ingreso a la educación superior. Revista de Salud Pública y Nutrición, 17(4), 41-47.

Editor: Esteban G. Ramos Peña, Dr. CS., Universidad Autónoma de Nuevo León, Facultad de Salud Pública y Nutrición, Monterrey Nuevo León, México. Copyright: (C)2018 Tijerina González LZ et al. This is an open-access article distributed under the terms of Creative Commons Attribution License [CC BY 4.0], which permits unrestricted use, distribution, and reproduction in any medium, provided the original author and source are credited.

Competing interests: The authors have declared that no competing interests exist.

DOI: https://doi.org/10.29105/respyn17.4-5

Recibido: 10 de octubre 2018;

Aceptado: 29 de noviembre 2018

Email: liliana.tijerinag@uanl.mx 


\title{
DEPRESIÓN, ANSIEDAD Y ESTRÉS EN ESTUDIANTES DE NUEVO INGRESO A LA EDUCACIÓN SUPERIOR.
}

\author{
Tijerina González Liliana Zandra*, González Guevara Erika*, Gómez Nava Marisol*, Cisneros Estala Martha \\ Aidee*, Rodríguez García Karla Yadira*, Ramos Peña Esteban Gilberto*.
}

*Universidad Autónoma de Nuevo León, Facultad de Salud Pública y Nutrición, México.

\section{RESUMEN}

Introducción: Muchos problemas de salud mental tienden a aparecer al final de la infancia o al comienzo de la adolescencia, según estudios más recientes, los problemas de salud mental constituyen la principal causa de morbilidad en los jóvenes. Objetivo: Determinar frecuencia de ansiedad, depresión y estrés en estudiantes de primer grado de una licenciatura en una universidad pública. Material y Métodos: Se realizó un estudio de corte transversal en una muestra de 520 estudiantes, utilizando el cuestionario autoadministrado DASS-21, que permitió establecer la prevalencia de depresión, estrés y ansiedad, El análisis estadístico se realizó mediante la prueba no paramétrica de independencia. Resultados: El promedio de edad de fue de 18.06 (DE \pm 1.82 ) años, el $80.8 \%$ de la población eran mujeres y un $19.2 \%$ eran hombres. El 19.9\% tiene algún grado de ansiedad, $36.9 \%$ algún grado de depresión y $19.8 \%$ de estrés. Conclusiones: Los componentes de la prueba DASS (depresión, ansiedad y estrés) están asociados, en el género femenino se encontró asociación depresión-ansiedad, ansiedad-estrés y depresión-ansiedad y en hombres solamente depresión-ansiedad.

Palabras Clave: Depresión, ansiedad, estrés, estudiantes universitarios.

\section{ABSTRACT}

Introduction: Many mental health problems tend to appear at the end of childhood at the beginning of adolescence, according to more recent studies, mental health problems are the main cause of morbidity in young people. Objective: To determine frequency of anxiety, depression and stress in first-graders of a bachelor's degree at a public university. Methods A crosssectional study was conducted in a sample of 520 students, using the self-administered questionnaire DASS-21, which allowed to establish the prevalence of depression, stress and anxiety. The statistical analysis was carried out using the nonparametric test of independence. Results: The average age was 18.06 (SD \pm 1.82 ) years, $80.8 \%$ of the population were women and $19.2 \%$ were men. $19.9 \%$ have some degree of anxiety, $36.9 \%$ some degree of depression and $19.8 \%$ stress. Conclusions: The components of the DASS test (depression, anxiety and stress) are associated, it was found association of depression-anxiety, anxiety-stress and depression-anxiety in women and in only depression-anxiety in men.

Key words: Depression, anxiety, stress, university students. 


\section{Introducción}

La salud mental se define como un estado de bienestar en el cual el individuo es consciente de sus propias capacidades, puede afrontar las tensiones normales de la vida, puede trabajar de forma productiva y fructífera y es capaz de hacer una contribución a su comunidad (OMS, 2013).

Es de importancia poder establecer la prevalencia de estudiantes con algún nivel de depresión, ansiedad, estrés al ingreso a educación superior a fin de establecer la salud mental y que pueda verse afectada por las exigencias, nivel de desarrollo y de competencias que establecen posibilidad de éxito o fracaso. (Gutiérrez Rodas, y otros, 2010).

La depresión se presenta como un conjunto de síntomas de predominio afectivo (tristeza patológica, apatía, desesperanza, decaimiento, irritabilidad, sensación subjetiva de malestar e impotencia frente a las exigencias de la vida) aunque, en mayor o menor grado, también están presentes síntomas de tipo cognitivo, volitivo y somático, por lo que podría hablarse de una afectación global psíquica y física, haciendo especial énfasis en la esfera afectiva (National Collaborating Centre for Mental Health, 2010).

El término ansiedad alude a la combinación de distintas manifestaciones físicas y mentales que no son atribuibles a peligros reales, sino que se manifiestan ya sea en forma de crisis o bien como un estado persistente y difuso, pudiendo llegar al pánico; no obstante, pueden estar presentes otras características neuróticas tales como síntomas obsesivos o histéricos que no dominan el cuadro clínico (Sierra, Ortega, \& Zubeidat, 2003).

El estrés es un estado persistente de sobre activación que refleja la dificultad constante de enfrentar las demandas de la vida, distinguiendo como consecuencia de este estado la baja tolerancia a la frustración. (Román Mella, Vinet, \& Alarcón Muñoz, 2014).

La depresión y la ansiedad son cuadros fenomenológicamente distintos, pero ha sido muy difícil distinguir entre ellos mediante las medidas clínicas o el uso de auto reportes, se ha sugerido que se debe a las escalas para medir la depresión y ansiedad que predominantemente miden el factor común de la afectividad negativa (Gurrola Peña, Balcázar Nava, Bonilla Muños, \& Virseda Heras, 2006).

En los últimos años, las investigaciones han dirigido sus esfuerzos hacia el estudio de la depresión y la ansiedad; en este sentido se ha realizado diversos estudios (Alonso et al., 2004; Beuke, Fischer, \& MCdowall, 2003; Brown, Campbell, Lehman, Grishman, \& Mancill, 2001; Dowrick et al., 1998; Michaud, Murria, \& Bloom, 2001), centrados en el análisis de factores como la edad, con el objetivo de determinar el inicio o mantenimiento de la depresión y la ansiedad; por lo anterior, muchos estudios se han centrado en la población universitaria, llegando a la conclusión de la alta prevalencia de la ansiedad y la depresión en esta población.

Se he observado un incremento notorio de manifestaciones depresivas particularmente en adolescentes femeninas respecto a periodos previos de la vida. (Román Mella, Vinet, \& Alarcón Muñoz, 2014). Según datos de la OMS cada año se suicidan más de 800,000 personas, y el suicidio es la segunda causa de muerte en el grupo de 15 a 29 años de edad, los trastornos mentales y el consumo nocivo de alcohol contribuyen a muchos suicidios (OMS).

La escala de Depresión, Ansiedad y Estrés (DASS21) fue diseñada para medir estados emocionales negativos de depresión, ansiedad y estrés; que incluye síntomas esenciales de cada condición y se excluye los que pueden estar presentes en ambos trastornos, por ejemplo, cambios de apetito. (Román, Santibáñez, \& Vinet , 2016). Originalmente constaba de 42 reactivos, esos reactivos fueron la base para conformar la versión corta que consta de 21 reactivos; posee subescalas específicas, la escala de la depresión mide la baja afectividad positiva, la escala de la ansiedad mide la agitación psicofisiológica y la de estrés, la afectividad negativa. (Gurrola Peña, Balcázar Nava, Bonilla Muños, \& Virseda Heras, 2006).

Según Antúnez y Vinet (2012), la discriminación perfecta entre medidas de auto - informes de ansiedad y depresión parece imposible ya que las correlaciones entre las escalas del DASS no se deberían únicamente a la carga de los factores, sino más bien a la continuidad natural entre los tres síndromes y, por ende, a la correlación esperable 
entre los factores, que puede surgir debido a que existen causas comunes subyacentes a la depresión, la ansiedad y al estrés.

En términos de calidad de vida, los trastornos de ansiedad y depresión afectan el desarrollo normal de los adolescentes, teniendo un impacto negativo en el rendimiento académico y en el desempeño psicosocial (Román Mella, Vinet, \& Alarcón Muñoz, 2014) (Meyer K., Ramírez F., \& Pérez V., 2013) Los estudiantes de Ciencias de la Salud pueden presentar elevados niveles de estrés, lo que se correlaciona con malos resultados académicos, adopción de hábitos poco saludables, y desarrollo de patologías mentales y físicas. (Meyer K., Ramírez F., \& Pérez V., 2013).

En adolescentes de 17 y 18 años, se ha encontrado una asociación significativa entre el grado de depresión y las tasas de síntomas depresivos posteriores, depresión mayor, desordenes de ansiedad, ideas suicidas e intentos de suicidas. (Fergusson, Horwood, Ridder, \& Beautrais, 2005).

Diversos estudios avalan que, en general, los estudiantes de carreras de la salud están expuestos a niveles de estrés más elevados que la población en general por sobrecarga académica, falta de tiempo para cumplir con obligaciones y realización de exámenes. (Castillo Pimienta, Chacón de la Cruz, \& Díaz-Véliz, 2016) (Marty, Lavín G., Figueroa M., Larraín de la C., \& Cruz M, 2005) De acuerdo a la literatura, para los estudiantes de carreras de la salud, el primer año es uno de los más estresante (Meyer K., Ramírez F., \& Pérez V., 2013) (Celis, Cabrera, Cabrera, Alarcón, \& Monge, 2001).

El Objetivo del estudio fue evaluar la presencia de depresión, ansiedad y estrés en estudiantes de nuevo ingreso a la educación superior, determinar si existe relación entre alguno de los tres componentes, así como comprobar si existe diferencias según género.

\section{Material y Métodos}

Se realizó un estudio de corte transversal, de tipo censal en 520 estudiantes de primer ingreso a la licenciatura en Nutrición, del semestre Enero-Junio 2018, para determinar la presencia de depresión, ansiedad y estrés, se utilizó la prueba DASS-21 versión en español de (Daza, Novy, Stanley, \& Averill, 2002); cada una de las tres escalas tiene siete ítems con formato de respuesta tipo Likert con cuatro alternativas, las que se ordenan en una escala de 0 a 3 puntos, el puntaje de cada escala se calcula con la suma de los puntajes de los ítems pertenecientes a esa escala y varía entre 0 a 21 puntos (Román Mella, Vinet, \& Alarcón Muñoz, 2014).

Una vez obtenido el puntaje para cada escala se clasificaron los niveles de depresión, ansiedad y estrés como: normal, bajo, moderado, severo y extremadamente severo; la e scala utilizada está construida para el DASS-42, por lo cual, para poder utilizar la escala para los resultados del DASS21 se deben multiplicar por dos (ver figura 1) (Lovibond \& Lovibond, 1995).

Figura 1. Escala para los puntajes DASS-42

\begin{tabular}{|lccc|}
\hline & Depresión & Ansiedad & Estrés \\
\hline Normal & $0-9$ & $0-7$ & $0-14$ \\
Bajo & $10-13$ & $8-9$ & $15-18$ \\
Moderado & $14-20$ & $10-14$ & $19-25$ \\
Severo & $21-27$ & $15-19$ & $26-33$ \\
Extremadamente severo & 28 o más & 20 o más & 34 o más \\
\hline (Lovibond \& Lovibond, 1995) & & &
\end{tabular}

Los estudiantes fueron informados de los propósitos y objetivos del estudio, y se les solicitó su participación voluntaria, después de esto se les entregó el cuestionario autoadministrado que garantizó la privacidad de los estudiantes y su participación anónima, cumpliendo con lo establecido por el Reglamento de la Ley General de Salud en Materia de Investigación para la Salud en caso de investigaciones con riesgo mínimo (Secretaria de Salud, 1984).

\section{Resultados}

El promedio de edad de los estudiantes fue de 18.06 (DE. \pm 1.82 ) años, el $80.76 \%$ de los estudiantes son del género femenino (n.420) y el $19.24 \%$ masculino (n.100).

El $19.42 \%$ de los estudiantes (n. 101) mostraron algún nivel de depresión, reportando a su vez que el $36.92 \%$ (n.192) presentaban algún nivel de ansiedad, en cuanto los niveles de estrés reportados se encontraron un $19.81 \%$ de los estudiantes (n. 103) presentaban niveles de estrés. (Tabla n. 1) 
Tabla 1. Niveles de Depresión, ensiedad y estrés en estudiantes* de nuevo ingreso a la educacion superior

\begin{tabular}{lcccccc}
\hline \multirow{2}{*}{ Niveles } & \multicolumn{2}{c}{ Depresión } & \multicolumn{2}{c}{ Ansiedad } & \multicolumn{2}{c}{ Estrés } \\
\hline Normal & $\mathrm{n}$ & $\%$ & $\mathrm{n}$ & $\%$ & $\mathrm{n}$ & $\%$ \\
Bajo & 419 & 80.6 & 328 & 63.1 & 417 & 80.2 \\
Moderado & 46 & 8.8 & 49 & 9.4 & 41 & 7.9 \\
Severo & 40 & 7.7 & 82 & 15.8 & 40 & 7.7 \\
Extremadamente & 8 & 1.5 & 27 & 5.2 & 19 & 3.7 \\
severo & 7 & 1.3 & 34 & 6.5 & 3 & 0.6 \\
\hline
\end{tabular}

Fuente: Encuesta

$* n=520$

En la combinación de depresión y ansiedad, el 42.9\% de los estudiantes presentaron combinaciones entre los niveles de depresión y ansiedad. El $1.5 \%$ presentaron nivel bajo, $2.7 \%$ moderado, $0.4 \%$ extremadamente severo tanto en depresión como en ansiedad. Los resultados de la prueba paramétrica demuestran asociación entre depresión y ansiedad. Tabla 2).

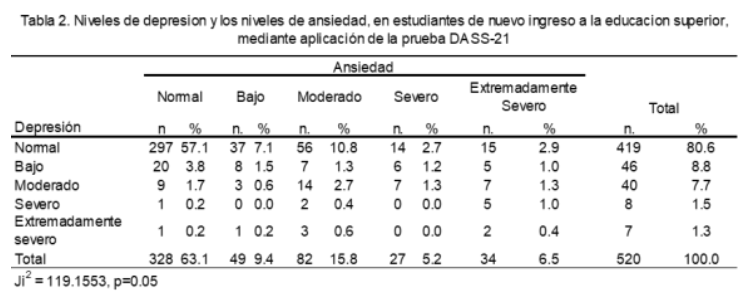

En cuanto a la presencia de estrés-ansiedad, el 40.6\% de los estudiantes presentaron combinaciones entre los niveles de esta combinación. El $2.3 \%$ presentaron nivel bajo, $1.3 \%$ moderado, $0.2 \%$ severo, $0.6 \%$ extremadamente severo tanto en depresión como en ansiedad. Los resultados de la prueba paramétrica demuestran asociación entre estrés y ansiedad (Tabla 3).

\begin{tabular}{|c|c|c|c|c|c|c|c|c|c|c|c|c|}
\hline \multirow[b]{3}{*}{ Estrés } & \multicolumn{10}{|c|}{ Ansiedad } & \multirow{2}{*}{\multicolumn{2}{|c|}{ Total }} \\
\hline & \multicolumn{2}{|c|}{ Nomal } & \multicolumn{2}{|c|}{ Bajo } & \multicolumn{2}{|c|}{ Moderado } & \multicolumn{2}{|c|}{ Severo } & \multicolumn{2}{|c|}{ Extremadamente } & & \\
\hline & $n$ & $\%$ & $n$ & $\%$ & $n$ & $\%$ & & $\%$ & $n$ & $\%$ & & $\%$ \\
\hline Normal & 309 & 59.4 & 37 & 7.1 & 55 & 10.6 & 12 & 2.3 & 4 & 0.8 & 417 & 80.2 \\
\hline Bajo & 12 & 2.3 & 4 & 0.8 & 14 & 2.7 & 8 & 1.5 & 3 & 0.6 & 41 & 7.9 \\
\hline Moderado & 7 & 1.3 & 6 & 1.2 & 7 & 1.3 & 6 & 1.2 & 14 & 2.7 & 40 & 7.7 \\
\hline Severo & 0 & 0.0 & 2 & 0.4 & 6 & 1.2 & 1 & 0.2 & 10 & 1.9 & 19 & 3.7 \\
\hline $\begin{array}{l}\text { Extremadamente } \\
\text { severo }\end{array}$ & 0 & 0.0 & 0 & 0.0 & 0 & 0.0 & 0 & 0.0 & 3 & 0.6 & 3 & 0.6 \\
\hline Total & 328 & 63.1 & 49 & 9.4 & 82 & 15.8 & 27 & 5.2 & 34 & 6.5 & 520 & 100.0 \\
\hline
\end{tabular}

En la combinación depresión-estrés, el 28.6\% de los estudiantes presentaron combinaciones entre los niveles de esta combinación. El $1.5 \%$ presentaron nivel bajo, $1.7 \%$ moderado, $0.4 \%$ severo, $0.6 \%$ tanto en depresión como en ansiedad. Los resultados de la prueba paramétrica demuestran asociación entre depresión y estrés (Tabla 4).

\begin{tabular}{|c|c|c|c|c|c|c|c|c|c|c|c|c|}
\hline \multirow[b]{3}{*}{ Depresión } & \multicolumn{10}{|c|}{ Estrés } & \multirow{2}{*}{\multicolumn{2}{|c|}{ Total }} \\
\hline & \multicolumn{2}{|c|}{ Normal } & \multicolumn{2}{|c|}{ Bajo } & \multicolumn{2}{|c|}{ Moderado } & \multicolumn{2}{|c|}{ Severo } & \multicolumn{2}{|c|}{ Extremadamente } & & \\
\hline & n. & $\%$ & & $\%$ & n. & $\%$ & & $\%$ & n. & $\%$ & $\mathrm{n}$. & $\%$ \\
\hline Nomal & 371 & 71.3 & 26 & 5.0 & 16 & 3.1 & 5 & 1.0 & 1 & 0.2 & 419 & 80.6 \\
\hline Bajo & 27 & 5.2 & 8 & 1.5 & 9 & 1.7 & 2 & 0.4 & 0 & 0.0 & 46 & 8.8 \\
\hline Moderado & 17 & 3.3 & 5 & 1.0 & 9 & 1.7 & 7 & 1.3 & 2 & 0.4 & 40 & 7.7 \\
\hline Severo & 1 & 0.2 & 0 & 0.0 & 5 & 1.0 & 2 & 0.4 & 0 & 0.0 & 8 & 1.5 \\
\hline $\begin{array}{l}\text { Extremadamente } \\
\text { severo }\end{array}$ & 1 & 0.2 & 2 & 0.4 & 1 & 0.2 & 3 & 0.6 & 0 & 0.0 & 7 & 1.3 \\
\hline Total & 417 & 80.2 & 41 & & 40 & 7.7 & & 3.7 & 3 & 0.6 & 520 & 100.0 \\
\hline
\end{tabular}

En cuanto a la combinación de depresión-ansiedad estratificado por sexo, el $29.4 \%$ de las mujeres tienen algún nivel en esta combinación, el 1.0\% presentaron nivel bajo, $2.5 \%$ moderado, $0.2 \%$ extremadamente severo tanto en depresión como en ansiedad. El $27.6 \%$ de los hombres tienen algún nivel en esta combinación, El $0.6 \%$ presentaron nivel bajo, $0.2 \%$ moderado, $0.2 \%$ extremadamente severo. Los resultados de la prueba paramétrica demuestran asociación entre depresión y estrés en ambos sexos (Tabla 5).

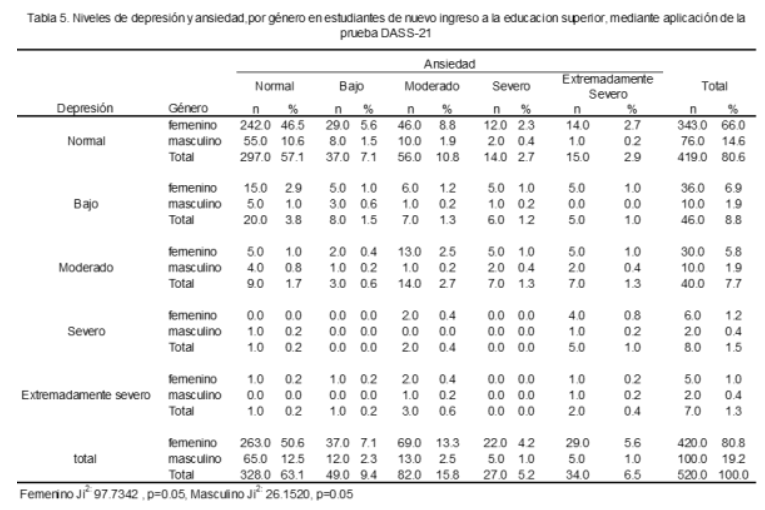

En la combinación de depresión-estrés estratificado por sexo, el $11.7 \%$ de las mujeres tienen algún nivel en esta combinación, el $1.5 \%$ presentaron nivel bajo, $1.3 \%$ moderado, $0.4 \%$ severo, tanto en depresión como en ansiedad. El 10.5\% de los hombres tienen algún nivel en esta combinación, El $0.4 \%$ presentaron nivel moderado. Los resultados de la prueba paramétrica demuestran asociación entre depresión y estrés en ambos sexos (Tabla 6). 


\begin{tabular}{|c|c|c|c|c|c|c|c|c|c|c|c|c|c|}
\hline \multirow[b]{3}{*}{ Depresión } & \multirow[b]{3}{*}{ Género } & \multicolumn{10}{|c|}{ Estrés } & \multirow{2}{*}{\multicolumn{2}{|c|}{ Total }} \\
\hline & & \multicolumn{2}{|c|}{ Normal } & \multicolumn{2}{|c|}{ Bajo } & \multicolumn{2}{|c|}{ Moderado } & \multicolumn{2}{|c|}{ Severo } & \multicolumn{2}{|c|}{$\begin{array}{l}\text { Extremadamente } \\
\text { Severo }\end{array}$} & & \\
\hline & & $n$ & $\%$ & $n$ & $\%$ & $n$ & $\%$ & $\mathrm{n}$ & $\%$ & $\mathrm{n}$ & $\%$ & $n$ & $\%$ \\
\hline & femenino & 303 & 58.3 & 19 & 3.7 & 15 & 2.9 & 5 & 1.0 & 1 & 0.2 & 343 & 66.0 \\
\hline \multirow{2}{*}{ Normal } & masculino & 68 & 13.1 & 7 & 1.3 & 1 & 0.2 & 0 & 0.0 & 0 & 0.0 & 76 & 14.6 \\
\hline & Total & 371 & 71.3 & 26 & 5.0 & 16 & 3.1 & 5 & 1.0 & 1 & 0.2 & 419 & 80.6 \\
\hline \multirow{3}{*}{ Bajo } & femenino & 19 & 3.7 & 8 & 1.5 & 8 & 1.5 & 1 & 0.2 & 0 & 0.0 & 36 & 6.9 \\
\hline & masculino & 8 & 1.5 & 0 & 0.0 & 1 & 0.2 & 1 & 0.2 & 0 & 0.0 & 10 & 1.9 \\
\hline & Total & 27 & 5.2 & 8 & 1.5 & 9 & 1.7 & 2 & 0.4 & 0 & 0.0 & 46 & 8.8 \\
\hline \multirow{3}{*}{ Moderado } & femenino & 13 & 2.5 & 2 & 0.4 & 7 & 1.3 & 7 & 1.3 & 1 & 0.2 & 30 & 5.8 \\
\hline & omasculino & 4 & 0.8 & 3 & 0.6 & 2 & 0.4 & 0 & 0.0 & 1 & 0.2 & 10 & 1.9 \\
\hline & Total & 17 & 3.3 & 5 & 1.0 & 9 & 1.7 & 7 & 1.3 & 2 & 0.4 & 40 & 7.7 \\
\hline \multirow{3}{*}{ Severo } & femenin & 0 & 0.0 & 0 & 0.0 & 4 & 0.8 & 2 & 0.4 & 0 & 0.0 & 6 & 1.2 \\
\hline & masculino & 1 & 0.2 & 0 & 0.0 & 1 & 0.2 & 0 & 0.0 & 0 & 0.0 & 2 & 0.4 \\
\hline & Total & 1 & 0.2 & 0 & 0.0 & 5 & 1.0 & 2 & 0.4 & 0 & 0.0 & 8 & 1.5 \\
\hline \multirow{3}{*}{$\begin{array}{l}\text { Extremad } \\
\text { amente } \\
\text { severo }\end{array}$} & femenino & 1 & 0.2 & 1 & 0.2 & 1 & 0.2 & 2 & 0.4 & 0 & 0.0 & 5 & 1.0 \\
\hline & masculino & 0 & 0.0 & 1 & 02 & 0 & 0.0 & 1 & 0.2 & 0 & 0.0 & 2 & 0.4 \\
\hline & Total & 1 & 0.2 & 2 & 0.4 & 1 & 0.2 & 3 & 0.6 & 0 & 0.0 & 7 & 1.3 \\
\hline \multirow{3}{*}{ total } & femenir & 336 & 64.6 & 30 & 5.8 & 35 & 6.7 & 17 & 3.3 & 2 & 0.4 & 420 & 80.8 \\
\hline & masculino & 81 & 15.6 & 11 & 2.1 & 5 & 1.0 & 2 & 0.4 & 1 & 0.2 & 100 & 19.2 \\
\hline & Total & 417 & 802 & 41 & 79 & 40 & 77 & 19 & 37 & 3 & 06 & 520 & 1000 \\
\hline
\end{tabular}

En cuanto a la combinación de depresión-ansiedad estratificado por sexo, el $6.1 \%$ de las mujeres tienen algún nivel en esta combinación, el $0.4 \%$ presentaron nivel bajo, $1.2 \%$ moderado, $0.2 \%$ severo, $0.38 \%$ extremadamente severo tanto en depresión como en ansiedad. El $4.6 \%$ de los hombres tienen algún nivel en esta combinación, El $0.4 \%$ presentaron nivel bajo, $1.2 \%$ moderado, $0.19 \%$ extremadamente severo. Los resultados de la prueba paramétrica demuestran asociación entre depresión y ansiedad estrés en ambos sexos (Tabla 7).

\begin{tabular}{|c|c|c|c|c|c|c|c|c|c|c|c|c|}
\hline \multirow[b]{3}{*}{ Ansiedad } & \multirow[b]{3}{*}{ Género } & \multicolumn{9}{|c|}{ Estrés } & \multirow{2}{*}{\multicolumn{2}{|c|}{ Total }} \\
\hline & & \multicolumn{2}{|c|}{ Normal } & \multicolumn{2}{|c|}{ Bajo } & \multicolumn{2}{|c|}{ Moderado } & \multirow{2}{*}{ Severo } & \multicolumn{2}{|c|}{$\begin{array}{l}\text { Exre madamente } \\
\text { Severo }\end{array}$} & & \\
\hline & & & & & $\%$ & & $\%$ & & $\pi$ & $\%$ & & $\%$ \\
\hline \multirow{3}{*}{ Normal } & femenino & 247 & 47.5 & 10 & 1.9 & 6 & 12 & $\begin{array}{lll}0 & 0.00\end{array}$ & 0 & 0.00 & 263 & 50.6 \\
\hline & masculino & 62 & 11.9 & 2 & 0.4 & 1 & 0.2 & $\begin{array}{ll}0 & 0.00\end{array}$ & 0 & 0.00 & 65 & 12.5 \\
\hline & Total & 309 & 59.4 & 12 & 2.3 & 7 & 1.3 & $\begin{array}{ll}0 & 0.00\end{array}$ & 0 & 0.00 & 328 & 63.1 \\
\hline \multirow{3}{*}{ Bajo } & femenir & 28 & 5.4 & 2 & 0.4 & 6 & 12 & $\begin{array}{ll}1 & 0.19\end{array}$ & 0 & 0.00 & 37 & 7.1 \\
\hline & & 9 & & 2 & 0.4 & 0 & 0.0 & $\begin{array}{ll}1 & 0.19\end{array}$ & 0 & 0.00 & 12 & 2.3 \\
\hline & Total & 37 & 7.1 & 4 & 0.8 & 6 & 12 & 20.38 & 0 & 0 & 49 & 9.4 \\
\hline \multirow{3}{*}{ Moderado } & femen & 46 & 8.8 & 11 & 2.1 & 6 & 12 & $\begin{array}{ll}6 & 1.15\end{array}$ & 0 & 0.00 & 69 & 13.3 \\
\hline & masculino & 9 & 1.7 & 3 & 0.6 & 1 & 0.2 & $\begin{array}{ll}0 & 0.00\end{array}$ & 0 & 0.00 & 13 & 2.5 \\
\hline & Total & 55 & 10.6 & 14 & 2.7 & 7 & 1.3 & $\begin{array}{ll}6 & 1.15\end{array}$ & 0 & 0.00 & 82 & 15.8 \\
\hline \multirow{3}{*}{ Severo } & femeni & 11 & 2.1 & & 1.0 & 5 & 1.0 & $\begin{array}{ll}1 & 0.19\end{array}$ & 0 & 0.0 & 22 & 42 \\
\hline & mascu & 1 & 02 & & 0.6 & 1 & 0.2 & $\begin{array}{ll}0 & 0.00\end{array}$ & 0 & 0.00 & 5 & 1.0 \\
\hline & Total & 12 & 2.3 & 8 & 1.5 & 6 & 12 & 10.19 & 0 & 0 & 27 & 5.2 \\
\hline \multirow{3}{*}{$\begin{array}{l}\text { Exrremadamente } \\
\text { severo }\end{array}$} & fen & 4 & 0.8 & 2 & 0.4 & 12 & 2.3 & 1.73 & 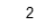 & 0 & 29 & 5.6 \\
\hline & mascu & 0 & 0.0 & 1 & 0.2 & 2 & 0.4 & $\begin{array}{ll}1 & 0.19\end{array}$ & 1 & 0.1 & 5 & 1.0 \\
\hline & Total & 4 & 0.8 & 3 & 0.6 & 14 & 2.7 & $10 \quad 1.92$ & 3 & 0.58 & 34 & 6.5 \\
\hline \multirow{3}{*}{ total } & femenino & 336 & 64.6 & 30 & 5.8 & 35 & 6.7 & 173 & 2 & 0.38 & 420 & 80.8 \\
\hline & masculino & 81 & 15.6 & 11 & 2.1 & 5 & 1.0 & $\begin{array}{ll}2 & 0.38\end{array}$ & 1 & 0.19 & 100 & 19.2 \\
\hline & Total & 380 & 80.2 & 37 & 7.9 & 34 & 7.7 & 173.65 & 3 & 0.58 & 520 & 100.0 \\
\hline
\end{tabular}

\section{Discusión}

En términos conceptuales, depresión y ansiedad son muy diferentes, pero la superposición clínica entre ambas condiciones ha sido reportada por clínicos y por investigadores; esta alta correlación puede explicarse por causas comunes de estados afectivos negativos. Por otra parte, los tres estados pueden aparecer frente a condiciones contextuales comunes que los activan (Antúnez \& Vinet, 2012) En la presente investigación se encontró que existe una asociación estadísticamente significativa entre los tres componentes, (depresión, ansiedad y estrés) $(\mathrm{p}<0.5)$.

Meyer, Ramirez y Perez (2013) Encontraron en su estudio que el $38.7 \%$ de los alumnos percibe niveles significativos de estrés y en el estudio de Román, Vinet y Alarcón (2014), un tercio de los adolescentes presenta síntomas depresivos clínicamente significativos, en este estudio se encontró que el $19.8 \%$ perciben algún nivel de estrés, aunque el porcentaje es menor $(p<.05)$ respecto a ambos estudios, pero el $36.92 \%$ de estos mismo estudiantes presentaban diversos niveles de ansiedad, no por eso se debe disminuir esfuerzos en la implantación de estrategias necesarias para el manejo precoz para el mejoramiento de la salud mental de estos estudiantes de manera que transcurran su estudio de licenciatura sin ese tipo de barreras.

El porcentaje de mujeres con estrés $(20.7 \%)$ en este estudio es mayor que el de los hombres $(\mathrm{p}<.05)$ lo cual concuerda con el estudio en Chile en estudiantes del área médica (Marty, Lavín G., Figueroa M., Larraín de la C., \& Cruz M, 2005).

En cuanto a la depresión, este estudio ha encontrado una prevalencia $(19.3 \%)$ mayor $(\mathrm{p}<.05)$ que en el estudio de en estudiantes de educación media, Cajigas, Kahan, Luzardo y Ugo (2010).

El padecimiento de trastornos de salud mental, que puede tener efectos importantes en la salud general y el desarrollo del adolescente, tiende a ir asociado a diversos problemas sanitarios y sociales, como un consumo más elevado de alcohol, tabaco y sustancias ilícitas, el embarazo, el abandono escolar y la asunción de conductas delictivas (Meyer K., Ramírez F., \& Pérez V., 2013) (OMS, 2018). Estudios centrados en la población universitaria evidencian que son altas las prevalencias en este tipo de trastorno tal como los realizados por Amézquita, González y Zuluaga, 2000; Gallagher, 2002 reportaron niveles de depresión y ansiedad de $41.7 \%$ a $58.1 \%$, valores 
que son superiores a los encontrados en nuestro estudio. En cuanto a los realizados por Campo-Cabal y Gutiérrez, 2001; Galli, 2005 y Miranda, Gutiérrez, Bernal y Escobar, 2000, reportaron de 30\% a $44 \%$ los valores de nuestro estudio son superiores en lo referente a la depresión y al estrés no así en cuanto a la depresión.

Los datos epidemiológicos del (Ministerio de la Protección Social, República de Colombia, 2006), señalan que la ansiedad y la depresión constituyen en Colombia un importante problema de salud, ocupando los primeros renglones en cuanto a los motivos de consulta psicológica y psiquiátrica, llegando a afectar al $17 \%$ de la población universitaria. (Agudelo Vélez, Casadiegos Garzón, \& Sánchez Ortíz, 2008), nuestros resultados son superiores en cuanto a ansiedad, pero, aun así, la prevalencia en la población de nuestro estudio es alta $19.3 \% \pm 3.4$ en depresión, $32.8 \pm 4.1$ en ansiedad y $16.5 \pm 3.4$ en estrés. Estos trastornos pueden actuar sobre variables de rendimiento académico, la deserción y el abandono y el bienestar emocional, entre otras. (Agudelo Vélez, Casadiegos Garzón, \& Sánchez Ortíz, 2008).

\section{Conclusiones}

Es importante destacar que la presente investigación se realizó a estudiantes que ya habían presentado su examen de admisión a la facultad y recibido la noticia que estaban oficialmente aceptado, se les aplicó la encuesta en los días en los días de inicio de cursos tiempo en que se les presentaron los planes de trabajo y lo cual no es motivo de estrés, aun así, se encontraron prevalencias altas en los trastornos de ansiedad, estrés y depresión en esta población estudiantil.

Todas las combinaciones de ansiedad, estrés y depresión se encuentran asociadas al género.

Es necesario desarrollar elementos de vigilancia en estudiantes ya que se ha demostrado la asociación entre estrés y los factores de exposición en clase, entrega de tareas y trabajos indicados en las unidades de aprendizaje, así como los exámenes obligatorios en estudiantes universitarios (Pozos, Preciado, Acosta, Aguilera, \& Delgado, 2014) de ahí que la realización de las pruebas de tamizaje de estrés al ingreso a educación superior es importante.

\section{Bibliografía}

Agudelo Vélez, D. M., Casadiegos Garzón , C. P., \& Sánchez Ortíz, D. L. (2008). CARACTERÍSTICAS DE ANSIEDAD Y DEPRESIÓN EN ESTUDIANTE SUNIVERSITARIOS. International Journal of Psychological research, 1(1), 34-39. Obtenido de http://www.redalyc.org/pdf/2990/299023503006.pdf

Antúnez, Z., \& Vinet, E. V. (2012). Escalas de Depresión, Ansiedad y Estrés (DASS - 21):Validación de la Versión Abreviada en Estudiantes Universitarios Chilenos. Terapia Psicológica, 30(3), 49-55. Obtenido de http://www.redalyc.org/html/785/78524692005/

Cajigas, S. N., Kahan, E., Mario, L., \& Ugo, M. d. (2010). Depresión en estudiantes de enseñanza media pública, severidad, género y edad. Ciencias Psicológicas, 4(2). Recuperado el 10 de Septiembre de 2018, de http://www.scielo.edu.uy/scielo.php?script=sci_arttex t\&pid=S1688-42212010000200003

Castillo Pimienta, C., Chacón de la Cruz, T., \& Díaz-Véliz, G. (Octubre-Diciembre de 2016). Ansiedad y fuentes de estrés académico en estudiantes de carreras de la salud. Investigación en Educación Médica, 5(20), 230 $237 . \quad$ Obtenido de http://www.redalyc.org/pdf/3497/349747925004.pdf

Celis, J., Cabrera, M., Cabrera, d., Alarcón, W., \& Monge, E. (2001). Ansiedad y estrés académico en estudiantes de medicina humana del primer y sexto año. Anales de la facultad de Medicina, 62(1), 25-30. Obtenido de http://www.redalyc.org/pdf/379/37962105.pdf

Daza, P., Novy, D. M., Stanley, M. A., \& Averill, P. (2002). The Depression Anxiety Stress Scale-21: Spanish Translation and Validation with a Hispanic Sample. Download PDF, 24(3), 195-205. doi:https://doi.org/10.1023/A:1016014818163

Fergusson, D. M., Horwood, L. J., Ridder, E. M., \& Beautrais, A. L. (JAn de 2005). Subthreshold depression in adolescence and mental health outcomes in adulthood. Arc Gen Psychiatry, 62(1), 66-72. Obtenido https://www.ncbi.nlm.nih.gov/pubmed/15630074

Gurrola Peña, G. M., Balcázar Nava, P., Bonilla Muños, M. P., \& Virseda Heras, J. A. (2006). Estructura Factorial y consistencia interna de la escala de Depresión, Ansiedad y Estrés (DASS-21) en una muestra no clínica. Psicología y Ciencia Social, 8(002), 3-7. Obtenido de http://www.redalyc.org/pdf/314/31480201.pdf

Gutiérrez Rodas, J. A., Montoya Vélez, L. P., Toro Isaza, B. E., Briñón Zapata, M. A., Rosas Restrepo, E., \& 
Salazar Quintero, L. E. (Enero-Junio de 2010). Depresión en estudiantes universitarios y su asociación con el estrés académico. Revista CES Medicina, 24(1), 7-17. Obtenido de http://www.redalyc.org/articulo.oa?id=261119491001

Lovibond, S. H., \& Lovibond, P. F. (1995). Manual for the Depresión Anxiety \& Stress Scales. Psychology Foundation(2nd). Recuperado el 07 de 2018, de https://serene.me.uk/tests/dass-score-guide.pdf

Marty, M., Lavín G., M., Figueroa M., M., Larraín de la C., D., \& Cruz M, C. (2005). Prevalencia de estrés en estudiantes del área de lasalud de la Universidad de los Andes y su relación con enfermedades infecciosas. Revista Chilena de neuropsiquiatría, 43(1), 25-32. Obtenido https://scielo.conicyt.cl/scielo.php?script=sci_arttext \&pid=S0717-92272005000100004

Meyer K., A., Ramírez F., L., \& Pérez V., C. (2013). Percepción de estrés en estudiantes chilenos de Medicina y Enfermería. Rev. Educ. Cienc Salud, 10(2), 79-85. Obtenido de http://www2.udec.cl/ofem/recs/anteriores/vol1022013 /artinv10213a.pdf

Ministerio de la Protección Social, República de Colombia. (2006). Un panorama nacional de la Salud y enfermedad mental de la salud en Colombia: Informe preliminar : Estudio Nacional de Salud Mental en Colombia. Obtenido de https://www.minsalud.gov.co/Documentos $\% 20 y \% 20$ Publicaciones/ESTUDIO\%20NACIONAL\%20DE\%2 OSALUD\%20MENTAL\%20EN\%20COLOMBIA.pdf

National Collaborating Centre for Mental Health. (2010). Depression. The treatment and managemente of depression in adults. London: The British Psychological Society. Obtenido de https://www.ncbi.nlm.nih.gov/books/NBK63748/

OMS. (Diciembre de 2013). Organización Mundial de la Salud . Recuperado el 27 de junio de 2018, de Salud mental: un estado de bienestar: http://www.who.int/features/factfiles/mental_health/e s/

OMS. (2018). Organización Mundial de la Salud. Recuperado el 27 de junio de 2018, de Salud de la madre, el recién nacido, del niño y del adolescente: http://www.who.int/maternal_child_adolescent/topics/ adolescence/mental_health/es/

OMS. (s.f.). Organización Mundial de la Salud. Recuperado el 27 de Junio de 2018, de 10 Datos sobre la

Salud http://www.who.int/features/factfiles/mental_health/m ental_health_facts/es/

Pozos, R. B., Preciado, S. M., Acosta, F. M., Aguilera, V. M., \& Delgado, G. D. (2014). Académic estress as a predictor of Chronic stress un univessity students. Psicología Educativa, 20, 47-52. doi:http://dx.doi.org/10.1016/j.pse.2014.05.006

Román Mella, F., Vinet, E. V., \& Alarcón Muñoz, A. M. (Agosto de 2014). Escala de Depresión, Ansiedad y Estrés (DASS-21): Adaptación y propiedades psicométricas en estudiantes secundarias de Temuco. Revista Argentna de Clínica Psicológica, XXIII, 179. $190 . \quad$ Obtenido de http://www.redalyc.org/articulo.oa?id=281943265009

Román, F., Santibáñez, P., \& Vinet , E. V. (2016). Uso de las Escalas de Depresión Ansiedad Estrés (DASS-21) como instrumento de tamizaje en jóvenes con problemas clínicos. Acta de Investigación Psicológica, 6(1), 2325-36. Obtenido de https://www.sciencedirect.com/science/article/pii/S20 07471916300539

Secretaria de Salud. (1984). Reglamento de la Ley General de Salud en Materia de Investigación para la Salud. México: Diario Oficial de la Federación. Obtenido de http://www.salud.gob.mx/unidades/cdi/nom/compi/rlg smis.html

Sierra, J. C., Ortega, V., \& Zubeidat, I. (2003). Ansiedad, angustia y estrés: tres conceptos a diferenciar. Revista Mal-estar e Subjetividade, 3(1), 10-59. Obtenido de http://www.redalyc.org/articulo.oa?id=27130102 